\title{
LA CASTELLANIZACIÓN DE LI LIVRES DOU TRESOR DE BRUNETTO LATINI EN LA CORTE DE SANCHO IV (1284-1295): ALGUNAS NOTAS SOBRE LA RECEPCIÓN DE LA ÉTICA ARISTOTÉLICA
}

\author{
THE TRANSLATION INTO CASTILIAN OF \\ BRUNETTO LATINI'S "LI LIVRES DOU TRESOR" \\ AT THE COURT OF SANCHO IV (1284-1295): \\ SOME REMARKS ON THE RECEPTION OF ARISTOTLE'S “NICOMACHEAN ETHICS”
}

\author{
ANA M. MONTERO \\ Saint Louis University, USA
}

\begin{abstract}
Resumen: Este trabajo se centra en la traducción al castellano de la obra de Brunetto Latini, Li Livres dou tresor. Dicha traducción -requerida por el rey castellano Sancho IV en torno a 1293permitió la aparición de la primera versión abreviada, en castellano, de la Ética a Nicómaco de Aristóteles (Summa Alexandrinorum); sorprendentemente la ética aristotélica no alcanzaría popularidad hasta el siglo XV. Este trabajo trata de ahondar en el tipo de recepción inicial que tuvo el Libro del tesoro, para lo cual se analizan las interpolaciones de breves explicaciones en la traducción al castellano. Se observa que el esfuerzo por aclarar el sentido de algunos conceptos se da mayoritariamente en la parte de la Ética a Nicómaco y afecta en particular a la noción de eudaimonía.
\end{abstract}

Palabras clave: Libro del tesoro; Sancho IV; Brunetto Latini; Etica a Nicómaco; Aristóteles; eudaimonía o felicidad.

\begin{abstract}
This article focuses on the translation into Castilian of Brunetto Latini's $L i$ Livres dou tresor. This translation was ordered by king Sancho IV around 1293, and included the first extant abridged version of Aristotle's Nicomachean Ethics (Summa Alexandrinorum) in Castilian. Nevertheless, the Aristotelian ethics would not become popular until the fifteenth century. This article aims at qualifying the initial reception of Libro del tesoro. Therefore, the interpolations of short explanations in the translation to Castilian are analyzed. It is observed that the effort to clarify the meaning of some concepts is evident in the chapters on ethics and does impinge on the Aristotelian notion of happiness or eudaimonia.
\end{abstract}

Keywords: Book of treasure, Sancho IV, Brunetto Latini, Nicomachean Ethics, Aristotle, eudaimonia or happiness.

\section{SUMARIO}

1. Contenido e importancia de Li Livres dou tresor de Brunetto Latini.- 2. Li Livres dou tresor de Brunetto Latini en las cortes de Alfonso X y Sancho IV: fuentes y carácter de la traducción.3. Libro del tesoro: interpolaciones e interpretación del concepto de eudaimonía aristotélica.Conclusión.- Indice A. Indice B. 


\section{CONTENIDO E IMPORTANCIA DE LI LIVRES DOU TRESOR DE BRUNETTO LATINI}

En un sensacionalista pasaje del Infierno en la Comedia de Dante Alighieri, éste sorprendía a su viejo maestro o guía intelectual, Brunetto Latini (1220-1294), en el círculo donde eran castigados los sodomitas. Y en el diálogo de este peculiar reencuentro, Latini manifestaba su aspiración a inmortalizarse gracias a los meritos de Li livres dou tresor, una compilación de saberes que había redactado en francés, durante su exilio entre 1261 y 1266, con el objetivo de estimular la regeneración política de Florencia: "sieti racommandanto il mio Tesoro/ nel qual io vivo ancora, e più non cheggio" (Inferno XV vv. 119-20). Sin duda Li livres dou tresor no decepcionó al público medieval; así lo testimonian los más de ochenta manuscritos en francés, los cincuenta y cuatro en italiano, quince en castellano, cuatro en catalán y uno en aragonés conocidos hoy en día (Baldwin, edición ii) ${ }^{1}$. Entre los factores que debieron propiciar el éxito de esta compilación de conocimientos no particularmente novedosos podemos señalar el carácter pragmático y secular del contenido, la rapidez y clara articulación con que Latini suele exponer las ideas (especialmente en el primer libro), y los recursos propagandísticos internos con los que se estimulaban la lectura y el aprendizaje. Uno de estos sugestivos recursos -el cual permite calar la naturaleza de este texto- es el procedimiento por el cual se organizan las materias sintetizadas en Li livres dou tresor: concretamente, Latini distribuye su material de acuerdo con las tres inquisiciones, matriz de la filosofía, que se planteó el ser humano en el momento en que intuyó la dignidad del entendimiento y ansió el conocimiento filosófico del que le había dotado Dios ${ }^{2}$. Esa intuición -que llevó a la humanidad a abandonar una etapa primitiva donde sólo se conocía "la ley de bestias" (I.2) - se manifestó primero en el esfuerzo por indagar

\footnotetext{
${ }^{1}$ Paolo Squillacioti - quien proporciona una completa recensión de los manuscritos europeos de Li Livres dou tresor - contabiliza hasta ciento y un manuscritos en francés, de los cuales 27 están incompletos o son fragmentarios. En italiano da un total de cincuenta y cuatro manuscritos, de los cuales veintiuno presentan el texto íntegro. "La tradizione manoscritta delle opere di Brunetto Latini", en Brunetto Latini. Tresor, Torino, 2007, xlvii-lix). En inglés, según J. Wittlin, sólo existe una traducción basada en un texto italiano (Llibre del tresor, vol I, Barcelona, 1971, p. 14); a pesar de esta escasa proyección, Rita Copeland estima que Li Livres dou Tresor influyó de manera notable en Confessio amantis de John Gower (Rhetoric. Hermeneutics, and Translation in the Middle Ages, Cambridge, 1991, pp. 209-210). En castellano, segun Paolo Squillacioti, existen quince manuscritos, de los cuales uno es simplemente un breve fragmento. Spurgeon Baldwin, en su edición del texto castellano, señala trece manuscritos ya que no incluye dos descubiertos por Julia Bolton Holloway: Span. D. I de la Bib. Bodleian en Oxford y Ottoboniano lat. 2054 de la Biblioteca del Vaticano (Cf. BALDWIN, Libro del tesoro, Madison 1989, vii, y SQUILLACIOTI, p. liii).

2"Filosofia es buscamiento verdadero de las cosas naturales \& de las divinales \& de las humanales tanto commo honbre puede dellas entender" (I.2.1; todas las citas procedentes del Libro del tesoro están tomadas de la edición de Spurgeon Baldwin, mientras no se señale lo contrario; para localizar una cita, normalmente indicamos el número del libro, el del capítulo y el del fragmento, como suelen hacer los editores del texto de Latini). En palabras de Giovanni Villani, contemporáneo de Brunetto Latini, éste fue agente de una obra civilizadora: "Fu mondano uomo... fue cominciatore e maestro in digrossare i Fiorentini, e farli scorti in bene parlare ["e ad insegnare loro l'eloquenza'], e in sapere guidare e reggere la nostra repubblica secondo la Politica" (Pietro G. BELTRAMI, Introduzione, Brunetto Latini. Tresor, Torino, 2007, p. IX).
} 
cuál era la naturaleza de lo terrenal y lo divino. Sintetiza Latini aquí nociones de teología, geografía y economía, destacando, en particular, una historia universal del trasvase de poder y un bestiario, con los que se configura el primer libro. Con la segunda inquisición -qué debe hacer el hombre y qué no- Latini pasa de la ciencia teórica a la práctica, la cual engloba la ética, la economía y la política, aunque en el libro se reduce sólo a un denso compendio ético de origen aristotélico. Finalmente, se desemboca en el tercer tema de indagación esencial para el progreso social - por qué debe hacer el hombre lo que hace o cómo debe justificarlo-, apartado que suscita el examen del arte de la retórica y del gobierno. En resumen, las materias se organizan por orden creciente de importancia y de acuerdo al esbozo de una historia del despertar del hombre a la civilización. Para Latini, la disciplina fundamental -empinada sobre los hombros de la retórica y englobando a todas las demásva a ser la política: "Por esto podedes entender que el arte que muestra \& enseña governar la çibdat es mayor et mas prinçipal \& señora de todas las artes, por que so ella se contienen muchas artes buenas \& honrradas" (II.3.1.), “ ...politica, que quiere dezir el governamiento de las çibdades que es la mas noble \& más alta sçiençia \& el más noble ofiçio que sea en tierra" (III.73.1). Además, en el título y prólogo de la obra, los saberes son equiparados a un tesoro, lo que hace más consciente al lector de la trascendencia y utilidad del texto que tiene entre manos. Así, el primer libro - es decir, la teología, la historia y las ciencias naturales - se identifican con monedas o "dineros contados"; la formación ética del segundo libro es vista como piedras preciosas, mientras que el libro tercero - la retórica y la política-son oro fino. Singularmente, en el proyecto educativo de Li livres dou tresor, la teología ya no es la reina de las ciencias. Brunetto Latini, como el mismo nos dice, no es ni teólogo ni clérigo; su sacramento de las relaciones humanas es el de la justicia, y aunque no abandona nunca el discurso religioso cristiano y el elogio a la iglesia, sus intereses se decantan abiertamente por materias necesarias para la convivencia social, como son la oratoria y la política ${ }^{3}$.

Finalmente, tampoco podemos ignorar que el libro en sí, como objeto material, fue concebido como un pequeño tesoro; en otras palabras, como valija diplomática con la que captar el interés de los hombres de poder -en el caso de su primer lector, Carlos de Anjou, el objetivo fue instruirlo en el modelo de organización política comunal de las ciudades italianas-, o como regalo con el que fortalecer relaciones que pudieron propiciar el intercambio de más textos. Es decir, no sólo esta obra facilitaba la formación del príncipe, o más concretamente del gobernador de una ciudad italiana, sino que - a veces acompañada de elaboradas iluminaciones- era símbolo de un poder basado en las ideas y en los medios económicos.

\footnotetext{
${ }^{3}$ Brunetto Latini altera su plan inicial; así incluye nociones de economía dentro del libro primero, dedicado a la filosofía teórica, y excluye el tratamiento de la lógica. Para un estudio Sobre la disparidad entre el proyecto inicial y el resultado, véanse las observaciones de Rita Copeland (op. cit., pp. 208-9).
} 
The Tresor is widely dispersed throughout Europe; copies are found in the Vatican, El Escorial, Leningrad, Oxford, Arras, Brussels, Paris, Naples, and elsewhere. (These are all cities with which the Florentine bankers had dealings.) The first version is a diplomatic presentation text, written for Charles of Anjou. But Brunetto was able to make generic copies of the Tresor and present them also to other major figures the Florentines wished to influence and impress, such as Alfonso el Sabio ${ }^{4}$.

Estas son pues las líneas maestras de una sugerente compilación de disciplinas, que vio la luz en Francia, aunque se estima que empezó a gestarse en España, durante la breve embajada de Latini en la corte de Alfonso $\mathrm{X}^{5}$. Latini buscaba el refuerzo de las tropas castellanas en el enfrentamiento entre güelfos y gibelinos, y a cambio ofrecía respaldar la coronación de Alfonso X como emperador, a la cual se oponían los gibelinos. Y aunque los propósitos políticos de Latini se vieron frustrados durante su regreso a Italia ese mismo año por la derrota de su bando - lo que le forzó a exiliarse en Francia- no debió pasar lo mismo con sus aspiraciones intelectuales, pues, como opina Julia Bolton Holloway (1985, p. 481), donde aparentemente triunfó su embajada fue en el orden literario.

Este encuentro entre Brunetto Latini y Alfonso $\mathrm{X}$ es el punto de partida de este trabajo, cuyo objetivo es el de ahondar en la recepción inicial que tuvo el Libro del tesoro, obra clave en el desarrollo de la filosofía moral en Castilla ya que facilitó la entrada de una primera versión, abreviada y en castellano, de la Etica a Nicómaco de Aristóteles. Tres momentos claves jalonan el itinerario del texto de Brunetto Latini en Castilla. El primero, el período de gestación de la obra, se produce a partir de 1260 , cuando las fuentes e hipotéticamente la ideología cultural alfonsíes dejan un cierto poso en el proyecto de instrucción del hombre de poder ideado por Brunetto Latini. Un segundo momento, el de la traducción de Li Livres dou tresor del francés al castellano - la cual fue encargada por Sancho IV, hijo de Alfonso X, al físico de su hijo, Alfonso de Paredes y a su escribano, Gómez Pascual en torno a 1293-, marca el camino de vuelta de esos saberes a la corte castellana. Finalmente, una tercera etapa clave para la recepción de esta obra es el siglo XV, época a la que pertenecen la mayoría de las copias existentes (todas excepto tres), dado que no han sobrevivido manuscritos originales del

${ }^{4}$ Cf. Julia Bolton Holloway, The Road Through Roncesvalles: Alfonsine Formation of Brunetto Latini and Dante -Diplomacy and Literature, en Emperor of Culture. Alfonso X the Learned of Castile and His Thirteenth-Century Renaissance, ed. Robert I. BURNS, Pennsylvania, 1990 , p. 116.

${ }^{5}$ Un aspecto relevante para entender la configuración y difusión inicial de Li Livres dou tresor afecta el entorno material en el que trabajaba Latini como exiliado en Francia, donde ejerció la función de notario para la población florentina asentada en París (BELTRAMI, op. cit., p. x). Holloway explica:

"Latini then went to France to live out six years of exile, thus absorbing not only Spanish culture but also French. He took up residence in the Lombard community in Arras in northern France using his notarial chambers for the production of encyclopedic books; these were written down by his students as he dictated to them in the French dialect of that region, the Picardian dialect of Artois. Many manuscripts of these texts ${ }_{2}$ often exquisitely illuminated, survive in European and American libraries" (Alfonso el Sabio, Brunetto Latini, and Dante Alighieri, "Thought", 60 (1985), p. 471). 
siglo XIII. El interés por Li Livres dou tresor en el siglo XV debió incrementarse gracias al auge que experimentó la ética aristotélica - la cual había sido reproducida de forma abreviada en el segundo libro del Libro del tesorodesde la segunda o tercera década del cuatrocientos ${ }^{6}$. Es entonces cuando tenemos por primera vez indicios concretos del impacto de Li livres dou tresor, ya sea mediante la constatación de la posesión de manuscritos - Martín el Humano, por ejemplo, poseyó cinco ejemplares de Li Livres dou tresor y el príncipe Carlos de Viana, dos (Labandeira Fernández 77) (Labandeira Fernández no debería ir a la nota a pie? $)^{7}-0$ mediante el aprovechamiento de su contenido. Así, Fernán Pérez de Guzmán (o al menos se le atribuyó a él) recopiló un centón de sentencias tomadas del Libro del tesoro, con el título de Floresta de filósofos. De varios dichos y sentencias políticas y morales y el escribano Pero Rodríguez de Lena - al levantar acta del Passo Honroso o gesta caballeresca de 1434 de Suero de Quiñones- interpoló fragmentos que también parecen proceder exclusivamente del libro II $^{8}$.

\section{LI LIVRES DOU TRESOR DE BRUNETTO LATINI EN LAS CORTES DE ALFONSO X Y SANCHO IV: FUENTES Y CARÁCTER DE LA TRADUCCIÓN}

Si volvemos brevemente al período inicial en la creación de Li livres dou tresor, - es decir, a la embajada que llevó a Latini a la corte castellana en $1260-{ }^{9}$, es fácil sospechar que Latini debió percibir con interés la labor puesta en marcha en los campos de la historia, las leyes, la filosofía natural y la literatura por el rey Alfonso X (1252-1284), un monarca de grandes inquietudes intelectuales, que había desplazado su radar cultural hacia lo islámico y que - con sus ojos puestos en los conocimientos legados desde la

${ }^{6}$ Los factores que contribuyeron a la propagación de la ética aristotélica dentro y fuera de los recintos universitarios fueron: la existencia de una nueva traducción de la Etica a cargo de Leonardo Bruni hecha en 1416-1417 - evento que provocó el conocido debate con Alonso de Cartagena sobre el carácter de la traducción y su instrumentalización en una sociedad cristianala frecuente reimpresión del texto de la Etica a Nicómaco, especialmente en el último cuarto de siglo, y el impulso que recibieron los estudios de la ética o moral aristotélica, como consta desde 1422 en la legislación académica de la universidad de Salamanca. Cf. A. PAGDEN, The Diffusion of Aristotle's Moral Philosophy in Spain, ca. 1400-ca. 1600, "Traditio" 31 (1975), pp. 287-313.

${ }^{7}$ El propio príncipe Carlos de Viana traduciría la ética de Aristóteles y propondría su armonización con el cristianismo en su "Carta exhortatoria a los letrados de España".

${ }^{8} \mathrm{Cf}$. Amancio LABANDEIRA FERNÁNDEZ, Un cronista español del siglo XV: entre la ciencia de Brunetto Latini y la nobleza de Suero de Quiñones, "Revista de Archivos, Bibliotecas y Museos", Madrid, LXXIX/1 (1976), pp. 78 y 87ss.

${ }^{9}$ Varios críticos - Amador de los Rios, Jaime Ferreiro Alemparte, Bolton Holloway y Fernando Gómez Redondo- han señalado que Li livres dou tresor se gestó en España. Sin embargo, para Pietro G. Beltrami se trata de una conjetura sin gran fundamento (op. cit., p. xi). En una de sus obras, Il Tesoretto, Brunetto Latini sugiere que la visita a España fue rauda: " $\mathrm{E}$ andai in ispangnia/ E feci l'ambasciata/ Che mi fue comandata;/ E pois sança sogiorno/ Ripresi mio ritorno" "Ed. Julia Bolton HOLLOWAY, New York \& London, 1981, versos 136-140; la cursiva es mía) y en una de las biografías sobre Latini, se ha señalado que Brunetto Latini no mostró ningún conocimiento de España en los textos conservados (Cf. Petrus A. MESSELAAR, Le vocabulaire des idées dans le "tresor" de Brunet Latin, Assen, 1963, p. 12). Sin embargo, la relación entre Latini y Alfonso X - ccomo se muestra más adelante- pudo prolongarse durante varios años y verse acompañada del intercambio de textos. 
antigüedad- lideró un espectacular renacimiento cultural que, de manera novedosa, afectaba principalmente las lenguas vernáculas. Cabe pues preguntarse qué pudo recabar Latini en los scriptoria del monarca castellano que le fuera útil en su posterior redacción de Li Livres dou tresor. En primer lugar, la Summa Alexandrinorum, o compendio de la Etica a Nicómaco de Aristóteles, la cual había sido traducida del árabe al latín apenas 20 años antes por Hermann Alemán —en 1243-4-y que, por su énfasis secundario en lo político, encajaba perfectamente en el diseño de Li Livres dou tresor ${ }^{10}$. Igualmente, Latini pudo adquirir copia del Almagesto, la obra de astronomía traducida del árabe en 1175 por Gerardo de Cremona, y citada como fuente en Li Livres dou tresor: "et las estrellas que podedes ver \& conosçer claramente en el firmamento son mill \& veynte \& dos segunt que fallan en el Almagesta" (I.110.2) $)^{11}$. Incluso existe la posibilidad de que Brunetto Latini se hubiera hecho con un manuscrito latino de la Política de Aristóteles, ya que, como nos recuerda Ferreiro Alemparte, es citada tanto en las Partidas de Alfonso como en Li livres dou tresor ${ }^{12}$.

${ }^{10}$ El libro II contiene un total de ciento treinta y un capítulos; los primeros cuarenta y nueve capítulos corresponden a la Summa Alexandrinorum. A partir del capítulo cincuenta, Latini recurre a las siguientes fuentes: Moralium Dogma Philosophorum de Guillermo de Conches, Doctrina de arte loquendi et tacendi de Albertano de Brescia, De quattour virtutibus cardinalibus sive Formula vitae honestae de Martino de Braga, Summa de virtutibus de Guillermo Peraldo y Sententiarum libri tres de Isidoro de Sevilla (BELTRAMI, op. cit., XVIII; WITTLIN, op. cit., vol I, p. 13).

Hay que considerar la posibilidad de otros dos "itinerarios" posibles - que excluyen a España - que pudieron permitir que la Summa Alexandrinorum - llegara a manos de Brunetto Latini. Primero, Brunetto Latini tuvo oportunidad de dar con la obra en París, dado que - como nos informa Marie Thérèse D'Alverny- consta que Hermann visitó París, estableció vínculos con la universidad y llevó allí su obra; de hecho, en 1250 Richard de Fournival ya había conseguido una copia de la Summa Alexandrinorum (el manuscrito latino 16581, de la Biblioteca Nacional de Paris), la cual además contiene el "liber qui dicitur Moralium dogma phylosoforum" (sic) "Remarques sur la tradition manuscrite de la "Summa Alexandrinorum", La transmission des textes philosophiques et scietifiques au Moyen Âge, ed. Charles BURNETT, Aldershot, 1994, pp. 266,269 y 270). Segundo, no se debería descartar la posibilidad de que Hermann Alemán y Brunetto Latini se hubieran conocido en Italia dado que Hermann - tras su residencia en Toledo (1240-1246) - se trasladó al sưr de Italia (1258-1266) a la corte de Manfredo, líder de los gibelinos y por lo tanto en oposición con el bando de Latini (Clara Foz, El traductor, la iglesia y el rey, Barcelona, 2000, p. 64).

Sería interesante investigar el tipo de traducción que hizo Brunetto Latini del compendio aristotélico árabe ya traducido al latín por Hermann; que yo sepa no existe ningún trabajo al respecto. Beltrami, de manera general, ,efine Li livre dou tresor como "un accorto montaggio di testi tradotti piú o meno liberamente" (op. cit., p. VIII; la cursiva es mía).

${ }^{11}$ Madeleine Dillay apunta que Brunetto Latini usa el Almagesto de Alfraganus traducido por Gerardo de Cremona (HollOWAY, 1985, op cit., p. 472), aunque se queja de lo distorsionado que aparece el contenido de esta obra en Li Livres dou tresor. Federico II en Italia había encargado otra traducción de la obra de Claudio Tolomeo en 1230.

${ }^{12}$ Dada la importancia de la política en la Etica a Nicómaco, cabe la posibilidad de que Latini se estuviera refiriendo a esta obra aristotélica y no a la Política. Ferreiro Alemparte, quien propone que Li livres dou tresor fue dedicado a Alfonso (Recepción de las Eticas y de la Política de Aristóteles en las Siete Partidas del Rey Sabio, "Glossae: Revista de Historia del Derecho Europeo", 1 (1988), p. 105), sugiere también que la curiosa referencia a las hijas de Adán y Eva fue tomada de la General Estoria, aunque matiza que pudo proceder de la fuente comun, la Historia scholastica de Pedro Comestor (Hermann el Alemán, traductor del siglo XIII en Toledo, "Hispania Sacra", XXXV (1983), p. 25) Igualmente, Ferreiro Alemparte estima que el siguiente pasaje "et nuestro enperador dice en el Libro de las Leys que començamiento es la mayor partida de la cosa" (12) encubre una referencia a Alfonso y a las Partidas (BALDWIN, op. cit., p. vi). Por otro lado se estima que Brunetto Latini actúo como puente cultural entre Alfonso X' y Dante, discípulo de Latini: "A number of scholars have asked whether Brunetto's embassy and exile might have transmitted from Alfonso to Brunetto's student Dante Alighieri a translation of The Ladder of Mahomet, a work that has striking parallels to Dante's La Divina Commedia. 


\begin{abstract}
Mais en ceste derraine partie vieut mestre Brunet Latin acomplir a son ami ce ke li avoit promis entour le commencement dou premier livre, la u il dist que son livre defineroit en politique, c'est a dire des governemens des cités, ki est la plus noble et la plus haute science et li plus nobles offices ki sois en tiere, selonc ce que Aristotles prueve en son livre. (Ferreiro Alemparte 1988 , p. 123).
\end{abstract}

En resumen, muy probablemente la aportación principal cedida por los scriptoria alfonsíes -aportación nada desdeñable- sería la ética aristotélica, en su forma abreviada, que Latini colocó en su segundo libro y que sería usada también de manera fragmentaria y sumaria en las Partidas del rey Sabio: "It is clear that Latini in the Tresor was taking from Alfonso el Sabio the 'treasure' of wisdom, Aristotle's Ethics, and was attempting to transmit this material to Charles of Anjou, to educate him as Aristotle had educated Alexander" ${ }^{13}$. Y aunque ignoramos cuándo y cómo se produjo este intercambio de textos, es posible que la relación entre Brunetto Latini y Alfonso se prolongara durante años, ya que Brunetto Latini debió enviar a Alfonso un ejemplar de Li Livre dou tresor (o quizás más de uno, puesto que hubo dos redacciones $)^{14}$. No sólo eso, de acuerdo con el juicio de Bolton Holloway, Il Tesoretto - otra obra de Latini, aunque de ésta no se han conservado copias o traducciones en España- formó también parte de algún tipo de correspondencia, testimonio de la admiración de Latini por Alfonso. El rey castellano, por su parte, también pudo regalar un ejemplar de las Cantigas de Santa María a Latini o al gobierno güelfo en el exilio al que representaba Latini ${ }^{15}$.

Unos treinta y tantos años después de la embajada de Latini o veintiséis años después de la composición de la obra en francés, Sancho, hijo

(Holloway, 1990, op. cit., p. 109; véase nota 1). Igualmente Bolton Holloway alega que la definición usada por Dante para el término "peregrino" (¿romero?) evoca la información de las Partidas.

En el orden de las ideas, la política cultural de Alfonso X pudo también ejercer una cierta influencia en Brunetto Latini, aunque aquí ya entramos en el terreno de la conjetura. Por ejemplo, la decisión de Latini de redactar su enciclopedia en francés pudo verse ratificada o reforzada por el impulso dado al çastellano y al gallego en el reinado alfonsí (aunque también influyera el hecho que su lector principal, Carlos de Anjou, no hubiera dominado el latín).

${ }^{13}$ Cf. Holloway, 1990, op. cit., p. 115. Alfonso de Cartagena menciona la presencia de algunos textos procedentes de la Eticas aristotélicas en las Partidas de Alfonso X (FERREIRO ALEMPARTE, 1988, op. cit., p. 97).

${ }^{14}$ El manuscrito Escorial L-II-3 de Li livres dou tresor (versión en francés del siglo XIII) presenta notas al margen en latín, escritas quizás por Latini para Alfonso X (HOLLOWAY, 1990, op. cit., p. 118). Sin embargo, este manuscrito -que presenta algunas interpolaciones cristianizantes - no fue usado para crear la traducción castellana. Hay otros dos manuscritos franceses en España: uno del siglo XIII en el Archivo diocesano de Barcelona, que es sólo un fragmento, y el manuscrito 5-I-6, del siglo XIV, en la Biblioteca Colombina de Sevilla.

15 "It is now clear that the Tesoretto or "little treasure" was originally written as a charming and witty diplomatic thank-you letter to Alfonso el Sabio, perhaps prefacing a translation into French or Italian of the Alfraganus-Ptolemy Almagest or of the entire Tresor (the dream vision breaks off just as Ptolemy is about to narrate to Latini all of his wisdom). The Tesoretto is preface to another text, some manuscripts stating that it is the Tesoro maggiore or "greater treasure." There is a reference to a Tesoretto having been in the library of the Marqués de Santillana, though it no longer seems to exist in that collection" (HOLLOWAY, 1990, op. cit., pp. 116-117). 
de Alfonso, mandó traducir Li livres dou tresor al castellano ${ }^{16}$. Con ello se da una peregrina coyuntura cultural: se recuperan los préstamos alfonsíes bajo una óptica cultural distinta $\mathrm{y}$, en consecuencia, surge la primera versión abreviada de la ética aristotélica en castellano; no sólo eso, el Libro del tesoro aporta también el primer bestiario conocido en castellano ${ }^{17}$, así como una descripción del sistema de gobierno de las ciudades italianas -muy diferente al de la monarquía castellana- cuyos principios, más proclives a favorecer los méritos propios que los heredados, pudieron despertar curiosidad, cuando no, actitudes de disconformidad ante el autoritarismo monárquico que ansiosamente buscó reforzar Sancho IV con su propia producción cultural ${ }^{18}$. Cabe pues preguntarse cuáles fueron los objetivos de Sancho al patrocinar esta traducción, que acabaría convirtiéndose en la obra del período sanchino de mayor difusión. Encontramos respuestas tanto en el ámbito cultural como en el político. Así, por un lado, con Sancho se produce un obvio alejamiento de las fuentes árabes -y con ello, de la astrología- y se favorece el acercamiento a las occidentales. Este giro cultural distintivo se debió ver estimulado por la necesidad política de conocer mejor las realidades francesa e italiana, dado que a Castilla le convenía bienquistarse y aliarse con Francia, país con el que finalmente lograría una alianza. Por otro lado, Sancho - encaramado al trono por medio de una guerra civil contra su padre- andaba necesitado de reivindicarse y regenerarse políticamente. El énfasis en las principales obras sanchinas - Lucidario y Castigos y documentos del rey Don Sancho (en adelante citada como Castigos) - por presentar a Sancho como un monarca providencial, incluso como figura mesiánica, es índice de esta preocupación; la obsesión por transmitir en castellano las pautas del comportamiento modélico que debe acatar un rey - como se hace en Castigos $^{19}$ - incide

\footnotetext{
${ }^{16}$ Según apunta Amador de los Ríos en el "Libro de cuentas de la casa de Don Sancho hallamos repetidamente mencionados los nombres de Maestre Alfon, fisico y Pero Gomez, escribano del rey, que tanto por sus oficios, como por otros conceptos perciben en los años de 1292 y 1293 algunas contías de maravedíses" (Historia crítica de la literatura española, vol III, Madrid, 1863, p. 21, nota 1). Aunque sólo poseemos los libros de cuentas desde diciembre de 1292 a noviembre de 1294 , es probable que fueran ésos los años en que se tradujo Li Livres dou tresor. También parecen ser éstos los anos cuando se impulsan las tareas culturales durante el reinado Sancho IV tras un período inicial de debilidad política.

${ }^{17}$ Es sorprendente que no se conozcan bestiarios procedentes de la corte de Alfonso X, lo que no indica que la información zoológica estuviera ausente. La presencia de animales en las iluminaciones en los libros de astrología del rey Sabio, así como la información tomada de los libros sobre animales de Aristóteles en el Lucidario de Sancho IV son indicio de un mundo activo en este terreno en las cortes castellanas de la segunda mitad del siglo XIII.

${ }^{18}$ En el momento de rebelión contra su padre Alfonso X y durante su campaña por captar adeptos, Sancho "garantizó la vuelta a los fueros y privilegios de los buenos tiempos pasados" a los concejos y el clero y prometió beneficios a los nobles (GONZÁLEZ JIMÉNEZ, Alfonso $X$ el Sabio, Barcelona, 2004, pp. 343-4). Una vez en el poder, quizás no sorprendentemente, Sancho IV disolvió las hermandades organizadas durante su rebelión y reclamó para sí un poder sin límites. Incluso antes de ser coronado rey, Sancho IV se vio como un elegido divino, como vicario de Dios, lo que dio a su poder un carácter incontestable [NIETO SORIA, Imágenes religiosas del rey, "En la España medieval", V (Madrid 1986), p.715-7].

${ }^{19}$ Castigos y documentos del rey don Sancho IV es el primer texto en castellano en el que se aborda la educación de un niño rey (Fernando, hijo de Sancho IV, probablemente contaba con seis $o$ siete años en el momento de la redacción). Fácilmente se puede argumentar que este texto - en la estela de otro libro de educación francés: Les propos de Saint Louis - no iba dirigido a niños, ni tenía mucho que ver con la educación primaria, sino que se trataba de una estrategia propagandística en tiempos de debilidad con las que intentar garantizar - por medio de una
} 
igualmente en este proyecto de regeneración política al que - pensamos- se adscribe en parte el Libro del tesoro, en particular su segunda parte. De hecho, se puede observar que en ambos textos se recurre a fuentes comunes, aunque su recreación sea claramente distinta. Este es el caso de Doctrina de arte loquendi et tacendi de Albertano de Brescia, que ocupa los capítulos 6167 del libro II, en Li Livres dou tresor (Beltrami, XIX).

[Capítulo 61: De guarda] Et si quisieres fablar, deves antes cuydar seys cosas: quien tu eres [+ 209: \& que es lo que quieres dezir, \& a quien] \& por que \& commo \& en qual tiempo, \& esto se entiende asi (Libro del tesoro II.61.4, pp. 130-131)

La boca del rey e del grand sennor mucho se deue guardar... En las palabras que ouieres a dezir mete mientes en ante que las digas en quantas cosas te agora diré, e así non errarás en ellas. Lo primero quién eres tú que las dizes, e de qué logar eres, o en quál logar estás de honrra. Lo segundo quál es el fecho sobre el que vienes a dezir aquellas palabras... Lo terçero quién es aquella persona a que las dizes... Lo quarto qué logar es aquél do las estás diziendo o ante quáles. Lo quinto... (Castigos 220) ${ }^{20}$.

El onbre callado es muy mas guardado en si. \& las palabras son semejantes a las saetas que onbre tira, que son ligeras de tirar, mas despues que son tiradas non las puede onbre retener (Libro del tesoro II.62.6, p. 132)

Mio fijo, para mientes e comide mucho sobre ello la palabra que dixeres ante que la digas. Ca tal es la palabra del omne desque sale por la boca commo la saeta de que sale de la ballesta que non la puede ninguno torrnar fasta que llega allí do ha de ferir. (Castigos 217) ${ }^{21}$

En cuanto al carácter de la traducción hecha, un cotejo entre el texto francés -editado por Chabaille, Carmody, Beltrami et al. respectivamentey la versión castellana permite constatar que predomina la voluntad de

estrategia sentimental basada en la exaltación del amor filial de Sancho IV - el traspaso de la corona a un príncipe que ni siquiera se encontraba legitimado por la iglesia.

${ }^{20}$ Todas las citas de Castigos proceden de la edición de Hugo Oscar BIZARRI, Castigos del rey don Sancho IV, Madrid, 2001.

${ }^{21}$ Se puede nencontrar otros testimonios similares donde se usan fuentes comunes con énfasis e intenciones distintas en ambas obras. Por ejemplo, tanto en Libro del tesoro como en Castigos, se explican detalladamente los comportamientos de las abejas, probablemente siguiendo las lecciones de San Isidoro en las Etimologías y la más contemporánea adaptación de Bartolomé Anglicus. Sin embargo, el carácter modêlico de las abejas se proyecta en direcciones diferentes. Asi, Latini - dentro de su bestiario en el libro I- resalta la solidaridad de las abejas ("et sabet que entre todas las animalias del mundo las abejas an en todos sus linages todas cosas mas comunales que las otras.... I.154.3) y señala que escogen por rey aquel que muestra naturalmente más nobleza. Sancho, por el contrario, recurre a ellas en Castigos dentro de un capítulo sobre el origen de la institución monárquica, con el fin de fortaleceer argumentos políticos más afines a su politica ("tu deues saber que la e[n]xambre de las abejas que fazen vn rey entre sí, e éste ẹs mayor e más fuerte e más hermoso que todas las otras abejas" X.40). Igualmente, el consejo aristotélico de no poner a hombres jóvenes en el poder por su falta de preparación -repetido por Latini en III.75.2, p. 218 y II.54.1 p. 126- se vierte con la misma cita en Castigos, pero con palabras diferentes: “Dize el rey Salamón: Ay de la tierra en que el rey es mozo de seso et de Sentido..." (Castigos 127); "Diz Salamon: por mal es la tierra que moços la rigen..." (II.54.2). En definitiva, aunque algunos han señalado la posibilidad de que Libro del tesoro fuera usada como fuente en Castigos, creo que es imposible probar una influencia textual directa - dadas las diferencias en la mentalidad de fondo y en la redacción-, pero sí es fácil argumentar que existía una comunión de intereses en torno a temas ético-políticos. 
recuperar de manera literal y fiel el texto de Latini; es decir, se siguió el método tradicional medieval de traducir palabra a palabra (verbum de verbo). Aunque no se ha facilitado todavía un stemma de los manuscritos castellanos conservados, a juicio de su editor, Baldwin, las catorce copias que señala en su edición derivan de una misma rama (como se demostrará más adelante). Sin embargo, se desconoce cuál fue el manuscrito francés que se usó para la traducción y, por lo tanto, las particularidades que pudo tener y que hubieran afectado el proceso de traducción ${ }^{22}$. Poco sabemos también de su público y como hemos afirmado, nada de su potencial impacto cultural, en apariencia, muy débil hasta el siglo XV. Estas lagunas nos fuerzan a prestar más atención a la traducción en sí, la cual puede parcialmente iluminar el tipo de acogida que recibió esta obra.

\section{LIBRO DEL TESORO: \\ INTERPOLACIONES E INTERPRETACIÓN DEL CONCEPTO DE EUDAIMONÍA ARISTOTÉLICA}

Es fundamentalmente en el terreno semántico donde encontramos algunas pistas sobre la recepción de esta obra en la corte de Sancho. Así es significativo que el traductor supla algunas explicaciones terminológicas para allanar la lectura de términos que podrían sorprender a los oyentes o lectores (idealmente, Sancho IV). Por ejemplo: "Parvificus que quiere decir mendigo"; "yracundus que quiere decir muy sañudo"; "artes mecánicas que son obras de mano"; "moralidad que es dicha de buenas costumbres"; "opinión que quiere decir asmança"; "furia que es dicha sandez", "tirano que quiere decir señor cruel"; "magnanimo es dicho onbre de grand coraçon"; etc. (véase índice A para una lista completa y su localización en las ediciones y los manuscritos) ${ }^{23}$.

${ }^{22}$ Es significativo que a partir del libro II, en la traducción al castellano, se omitan las referencias a Brunetto Latini; igualmente se omite "je dis" - usado por Latini probablemente mientras dictaba el texto a sus estudiantes o copistas - lo que excluye la vọz del maestro. Cabe pues sospechar que se hubiera iniciado la traducción de esta obra en el reinado de Alfonso X y completado en la época sanchina, cuanḍo el recuerdo del embajador Latini se hubiera difuminado. Esto justificaría que en varios manuscritos -Academia de la Lengua, 209; Biblioteca del Palacio, 3011; Biblioteca Nacional, 3380; y Universidad de Salamanca (1811 y 1697) - se atribuya la obra a Alfonso (Bolton HoLLOWAY, 1990. op. cit., p. 122), aunque hoy se estime que fue Sancho IV quien finalmente auspició la traducción.

${ }^{23} \mathrm{El}$ editor Spurgeon Baldwin consigna estas interpolaciones explicativas entre corchetes en su edición, indicando que aunque no hay paralelo en ninguno de los textos franceses citados por Chabaille y Carmody, queda la posibilidad de que lo que vemos en castellano represente una tradición textual válida" (op. cit., p. VIII). El hecho de que no aparezcan en al menos dos tercios de los manuscritos franceses (los revisados por Francis Carmody), ni en la reciente edición de Beltriami et al. - en la cual se hace una puesta a punto del texto francés- y de que resulten lógicas y útiles dentro de la traducción castellana permite deducir que se trata de una pequeña aclaración interpolada por el traductor de Sanchọ IV con el fin de orientar al lector con el vocabulario. La atención al vocabulario y al contenido de Li livre dou tresor se confirma en otros pasajes claramente manipulados por el traductor. Por ejemplo, la referencia a que España fue conquistada por Carlomagno en I.89.5 es omitida en el texto castellano.(p. 41 en la edición de Baldwin), quizás apoyándose en el testimonio del franciscano Juan Gil de Zamora, tutor de Sancho IV, quien en su De preconiis hispanie negaba la veracidad de tal aserto. Igualmente, el apunte de que el término francés "cesternas" recibe en España el nombre de "aljibes" (II.126.5, 
Sorprende que este esfuerzo por desbrozar el vocabulario, por allanarlo - que afecta a unos veinte términos-, no se produzca en el último libro, donde algunas vacilaciones con los términos políticos de las comunas italianas hubieran hecho más que necesario este apoyo semántico. Había sido éste el libro más valorado por Latini, dado que todo su proyecto educativo iba dirigido a familiarizar a los hombres de poder con los entresijos del gobierno en las ciudades italianas. Sin embargo, no debió despertar el interés entre los lectores castellanos, dadas las vacilaciones con el vocabulario y el poco esfuerzo por aclararlo (véase el apéndice B con la lista de términos). Por el contrario, las interpolaciones explicativas se concentran en el segundo libro (todas excepto dos) y la inmensa mayoría aparecen dentro del compendio de la Etica aristotélica (es decir, dentro de los primeros 49 capítulos). Más concretamente, más de la mitad de ellas aparecen a partir de la página 95, en la edición de Baldwin, es decir, tras la introducción a la obra, cuando - después de mencionar la importancia del arte de gobernar la ciudad, los tres tipos de vidas del ser humano, las tres facultades del alma, el bien y la felicidad - se habla de las virtudes de manera general y se elabora el concepto de eudaimonía aristotélica (capítulos 9 al 23, aproximadamente). También un gran número - como demuestra una revisión del Diccionario de Covarrubias - corresponden a términos de origen latino (poeta, opinión, iracundo, elección, doctrina, perpetuidad,...). Significativamente, términos como "intellectuel" no suelen ser traducidos nunca. Se buscaba - como es lógico pensar - la divulgación de la ética entre un público profano, ante el cual era necesario allanar el vocabulario de origen latino. Es también digno de mencionar que algunos de los términos explicitados son básicos para entender la argumentación aristotélica - como son: hábito, pasión, elección, relativo y beatitud o felicidad-y que no siempre son correctamente entendidos. Así, la coletilla añadida en castellano a la noción de "pasión" limita este término al sentido de "sufrimiento", mientras que su acepción dentro de la obra aristotélica es más amplia, incluyendo los significados de "sentimiento" o "apetencia, ira, miedo, atrevimiento, envidia, alegría, amor, odio, deseo, celos, compasión, y en general los afectos que van acompañados de placer o dolor" 24 .

Las interpolaciones explicativas son indicio de una curiosidad especial por esta parte del libro que, dado el predominio de textos didácticomorales en Castilla - desde el Calila et Dimna hasta los "Castigos del rey Mentón" en el Libro del caballero Zifar-, quizás no tenga nada de peculiar. Sin embargo, esta atención a la ética aristotélica intriga cuando constatamos que la obra moral del estagirita prácticamente es ignorada en el siglo XIII, el XIV y comienzos del XV. Pese a que es en España donde se traduce esta obra del árabe al latín por primera vez - la traducción de Hermann en 1240 precede a la más divulgada de Roberto Grosseteste, hecha del griego en 1246-

p. 67 en la edición de Baldwin) sólo aparece en los manuscritos españoles.

${ }^{24}$ Eds. Julián MARÍAS y María ARAuJo, Etica a Nicómaco, Madrid, 1949-2002, p. 24. Cf. MESSELAAR, op. cit., p. 67. 
1247 y revisada por Guillermo de Moerbeke entre 1250-1260_, pese a la atención prestada a todo lo aristotélico hasta el punto de que se extenderá la noción de un Aristóteles español, y pese a la reputación de Aristóteles como educador del hombre de poder, la Ética aristotélica no se va a cultivar en el ámbito de las universidades castellanas, ni aparentemente va a atraer la atención de los nobles españoles hasta el siglo XV. Este desinterés resulta insólito, dado que el texto existía y que en la universidad parisina fue estudiado y comentado desde muy temprano en el siglo XIII ${ }^{25}$. La propia traducción al castellano nos proporciona una pista. En efecto, uno de los casos más significativos en el esfuerzo por aclarar términos, afecta a un concepto clave en el sistema de pensamiento aristotélico. Se trata de la noción de felicidad aristotélica, que con frecuencia va acompañada en castellano de una interpolación la cual lo desvía de su sentido original y lo orienta hacia el terreno teológico o de trascendencia cristiana. Así, felicidad, o beatitudo, en el texto castellano van seguidos de la coletilla "beatitudo, que es la bienandança que nunca ha de fallecer" o "felicidad que es la bien andanza perdurable"; esta interpolación aparece algo más de una decena de veces en la traducción de la ética aristotélica (véase el índice A).

La felicidad es un concepto clave en el texto original aristotélico, hasta el punto de que uno de los editores en el siglo XX señala que el tema de la Etica a Nicómaco gira en buena parte en torno al esclarecimiento de la verdadera significación de dicha noción ${ }^{26}$. Así se señala también en la obra de Latini: "Donques trestoute l'entention de nostre livre est entor delit" (II. 12.1; en la edición de Carmody, p. 183); sin embargo, en la versión castellana se traduce esta frase al revés: "Et por ende toda la entençión de nuestro libro es contra deleyte" (II.12.1, Baldwin, p. 98; la cursiva es mía), lo que refleja la inicial dificultad con que se acogió la noción de felicidad aristotélica ${ }^{27}$.

\footnotetext{
${ }^{25}$ Nos atenemos en este punto a los estudios de Carlos Heusch y A. Pagden. Según Heusch, sorprende que no se recoja legislación alguna en universidaḍes españoles ạcerca dêl estudio de la Etica aristotélica hasta 1422 (Salamanca), aunque en la universidad parisina sí se encuentra la ética aristotélica en los estatutos en 1216 (89-90,92). Para explicar esta disparidad, Heusch alega dos posibles causas: 1) el riesgo de heterodoxia de la Etica aristotélica; 2) la preferencia, debido a las circunstancias culturales españolas, por la tendencia didáctico-moral orientalista, la cual representaba un sentido diferente de la moral al aristotélico [Entre didactismo y heterodoxia: Vicisitudes del estudio de la Etica aristotélica en la España Escolástica (siglos XIII y XIV), "La Corónica", 19/2 (1990-1), pp. 89-99].

${ }^{26} \mathrm{Cf}$. MARÍAS, op. cit., p. 3. Petrus A. Messelaar en p. 3 define "beatitude" no sólo como felicidad o dicha (felicité, bonheur), sino también como la alegría que resulta de la búsqueda de bien moral por sí mismo ("joie résultant de la recherçhe du bien moral pour lui-même"). En el texto frances de Brunetto Latini se lee: "Bien par lui est beatitude, ki est notre fin a quoi nos entendons» (II.5.1) ; "felicité es oevre de vertu, est il bien digne chose... felicitez est la fermeté et la constance des oevres propres de vertu» (III.47.4). En el texto moderno de Aristóteles se lee: "llamamos más perfecto al [bien] que se persigue por sí mismo que al que se persigue por otră cosa... tal parece ser eminentemente la felicidad, pues la elegimos siempre por ella misma" (MARÍAS, op. cit., p. 7).

${ }^{27}$ A este caso, habría que añadir algún "error" de traducción: así "beatitude" del francés es traducido como "divinal poderío":

"Mais de la beatitude ne se sovient ele [la memoire] par ymagination comme des autres, mais par lui meisme" (I.16.2).

"Mas del divinal poderio non se miembra ella [la memoria] por ymagen assy commo de las otras cosas, mas por sy mesma" (I.16.2; p. 19).

Baldwin también señala que, hablando de la definición de la "bienandanza", se traduce erróneamente "la chose au monde ki est trés millour \& trés joiouse et trés delitable" como: "la
} 
Latini había proclamado una felicidad de corte aristotélico basada en la nobleza del intelecto humano según la cual: "los mejores deleytes son fallados en filosofía, por el estudio de eternidat \& por la sotileza de verdat que son fallados en sus obras" (II.47.4; Baldwin, p. 121) ${ }^{28}$. La vida más noble -incluso por encima de la del legista o la del político- era la de la consagración al saber ("cada uno a noble vida \& muy digna por entendimiento": II.47.9; Baldwin, p. 122). Sin embargo, tras la condena de tesis naturalistas en el París de 1277 - donde se habían estigmatizado afirmaciones como que la vida más feliz era la del filósofo, que la felicidad pertenece a este mundo y no al otro, o que Dios no puede infundir la felicidad directamente- ${ }^{29}$ este tipo de exaltación filosófica podía emanar un vago tufillo heterodoxo. Más concretamente, en la corte castellana, donde el clero había ascendido al poder y se había responsabilizado de la producción cultural en la época de Sancho IV, este concepto aristotélico y otros podían congeniar con cierta dificultad con los preceptos cristianos. Sin duda, la coletilla castellana con la connotación de perdurabilidad, pretende fortalecer un concepto de felicidad distinto, más dependiente de una visión religiosa, donde bien andante o bienaventurado es aquel que pone sus ojos en los bienes del otro mundo. Para Tomás de Aquino, -quien se encargó de integrar el concepto aristotélico de felicidad dentro del contexto teológico cristiano- quedó claro que "the happiness of the life to come lies beyond any inquiry of the reason" y que "perfect human happiness is reserved for the life to come" (Kretzmann (ed), 678). Esta es la concepción que encontramos explícita en obras de Sancho IV, especialmente en Castigos $^{30}$ :

mas durable cosa que sea” (p. 95, nota 20). Y en la misma línea, se cristianiza un párrafo sobre la prosperidad:

"Lors te besoigne consaus quant tu as vie de prosperité, et ensi te remandra ta prosperité, et en bon leu fermement. Ne te muet trop hastivement, mais garde le leu u tu dois aler et jusc'a ou" (II.58.8).

"Mientras estas en buen andanca consejate commo podras aver la vida

perdurable \& asi te manterna dios en la tu buen andança \& en buen logar.

${ }^{28} \mathrm{En}$ el original francés leemos:

"La plus perfecte oevre, et la plus delitable ki soit, est felicité; més li trés millour delit sont trovées en philosophie, por la sollicitude de eternité et pour la soutillance de verité, ki sont troves en ses oevres. Et li dẹlit des sciences est plus savourous et plus delitable au sachant que a ciaus ki le vont querant... Donques la plus delitable chose ki en l'omme soit naturelement si est le oevre de intellec" (CARMODY, p. 220-1).

"Sapience est la dignité et l'avantage de l'ome en son mestier... sapience est felicité ke l'en doit eslire por lui, non pas comme chose ki amaine santé, mais comme santé meismes" (CARMODY, p.201).

${ }^{29} \mathrm{Cf}$. Norman KRETZMANN ET AL. (eds), The reception and interpretation of Aristotle's Ethics, en The Cambridge History of Later Medieval Philosophy, Cambridge, 1982-2003. p. 663.

La Etica aristotélica no sufrió el tipo de condenas que experimentaron los textos aristotélicos sobre filosofía natural. SSin embargo la interpretación de la eudaimonía sí generó controversia entre los pensadores cristianos, dado que se concebía la felicidad como un logro humano, independiente de valores absolutos o supremos. Para esta cuestión, véase tambiên el artículo Happiness: the perfection of man, editado por Kretzmann.

${ }^{30} \mathrm{La}$ sensibilidad con la que se vio este tema alcanza el siglo XV. Así en un popular compendio anónimo de la ética aristotélica - el cual tiene forma de texto escolar pero va dirigido al puablico laico cristiano- nos encontramos con un problema muy similar en la definición de la eudaimonía aristotélica:

"He [the author] describes the aim of the Ethics as the definition of 
E por esto han saluaçion las almas de los padres santos cristianos sieruos de Dios, los quales despreçiaron lo deste mundo quebrantando sus carnes, apremiandolas con grandes abstinençias por tal de non conssentir la uoluntad de la carne nin sabores e deleytes mundanales. E por esso les dio el Sennor la folgança de la su gloria del parayso, que dura e durara para siempre

... tal commo este es llamado bienauenturado. Esta buena andança es aquella que nunca ha de ser perdida (48-9).

Bien andante es aquel a que Dios da carrera e la el sabe tomar e vsa bien por ella, que auiendo los bienes deste mundo en guisa vse dellos por que non pierda los bienes del otro (195).

Por otro lado, se puede sospechar que, a finales del siglo XIII, la idea de un gobernador que trabaja por la felicidad de sus ciudadanos - como pide Aristóteles- tendría mucho de revolucionario en una Castilla que había sido víctima de una guerra civil (1282-1284): "asi deve el governador de la çibdat velar \& estudiar que pueda aprovechar a la çibdat, que a de guardar la filiçidat que conviene al alma [ + fr. entelllectuel et] les deve amonestar que fagan obras de virtudes, por que frutos dellas sean filiçidat"(II.7.8).

\section{CONCLUSIÓN}

Concluyendo, la traducción de Li Livres dou tresor al castellano ordenada por Sancho IV - ya fuera continuando una hipotética traducción iniciada en la corte alfonsí, o ya fuera por iniciativa propia- constituye una efeméride cultural de primer orden. Con el Libro del tesoro se recuperaba parte del legado aristotélico en filosofía moral ya recibido por la corte castellana alfonsí. Será esta parte (el libro II de Li Livres dou tresor) la que atraiga la atención de sus lectores, tanto en el siglo XIII como en el XV. Así lo testimonia desde el principio la atención al vocabulario - que sólo se prodiga en esta segunda parte por medio de interpolaciones explicativas-, y que refleja un primer intento, por parte de un público profano, de absorber los principios éticos aristotélicos. Sin embargo, la desviación semántica que se aplica a la noción de eudaimonía aristotélica, noción de difícil aceptación en el contexto hipercristiano de la corte de Sancho IV, nos lleva a pensar que se vio con cierta reticencia el contenido de la Etica a Nicómaco. A pesar de esta inicial dificultad, será muy probablemente la crisis económica y cultural del siglo XIV la que postergue la definitiva penetración de la filosofía moral aristotélica en las universidades y en las bibliotecas de los nobles.

\footnotetext{
"bienaventurança", a term which is hardly an accurate rendering of Aristotle's eudemonia, being closer to blessedness than happiness. Having made this step from an entirely secular concept to a Christian religious one, he goes on to suggest that happiness may be achieved in the afterlife, a point which Aristotle only mentions in passing, and then with an entirely different emphasis" (PAGDEN, op. cit., p. 301).
} 


\section{INDICE A}

Explicaciones de vocabulario añadidas en los manuscritos castellanos:

Gracias a la edición de M. Nieves Sánchez basada en los tres manuscritos conservados en la Universidad de Salamanca ${ }^{31}$, hemos podido comprobar que las interpolaciones explicativas, cuya lista proporcionamos a continuación, se encuentran también, sin excepciones, en los manuscritos de Salamanca. Por nuestra cuenta hemos verificado si se encuentra en el manuscrito 59-2-60 de la Biblioteca Colombina en Sevilla $^{32}$. En tercer lugar hemos revisado la edición del Spurgeon Baldwin (Madison 1989) aunque éste no marca si ese sintagma añadido aparece en todos los manuscritos castellanos o sólo en el que usa como manuscrito base (BNM 658). En cuanto a las ediciones de francés, nos hemos apoyado en la de Carmody, así como en la editada por Pietro G. Beltrami et al. (Torino, 2007) que - acompañando a la primera versión integral italiana en la época moderna- proporciona una puesta a punto del texto francés. seguido de:

El sintagma añadido en los manuscritos castellanos aparece en negrita,

1. Su localización general en el texto (libro, capítulo, párrafo); columna)

2. Su localización en la edición de Spurgeon Baldwin (Baldwin, página, folio).

3. Su localización en el manuscrito de la Biblioteca Colombina (Colombina,

Los términos con interpolaciones aparecen no alfabéticamente, sino según su orden de aparición en el texto.

Libro 1

-"gramatica... que nos enseña a fablar \& a escrivir... syn yerro de barbarismo \& solicismo. Et barbarismo es yerro que es fecho en sylaba o en una diçion, asy commo dize alguno "domínus" por "dóminus" et solicismo es yerro que acaesçe en ayuntamiento de dos partes, commo quien dize "buena onbre" (I.4.7; Baldwin 14a; Colombina 5vb).

- "ylem que quiere dezir la materia de que fueron criadas todas las cosas" (I.7.1; Baldwin 15a; Colombina 6ra).

Libro 2

-"beatitudo que es la bien andança que nunca ha de fallecer" (II.5.1; Baldwin 95a; Colombina 32va); "beatitude" es traducido como "la bien andança que

${ }^{31}$ Cfr. M. Nieves SÁNChEZ GONZÁLEZ DE HERRERo, El Libro del Tesoro de Brunetto Latini, Vigo, 2008. Holloway afirma que estos manuscritos no se originaron en Salamanca, sino que fueron trasladados de la colección real a la universidad, como parte de una donación hecha por Napoleón Bonaparte (1990: nota 55, p. 246; SÁNCHEZ, nota 1, p. 25).

${ }^{32}$ Puesto que éste manuscrito es uno de los pocos que Spurgeon Baldwin no pudo revisar durante la preparación de la edición, era importante trabajar con él. Se trata de una copia de finales del XIV, restaurada e incompleta, que consta de 70 folios a dos columnas. Tras el indice (folios $2 \mathrm{r}-4 \mathrm{v}$ ) vienen en el folio 4v sendas anotaciones de Bartolomé José Gallardo -quien el 5 de julio de 1809 escribe que la obra es atribuida generalmente al Sabio Rey D. Alonso X-y de Menéndez y Pelayo, quiẹn confirma que se tradujo al castellano por orden de Sancho IV. El incipit se lee: "Aquí comienza este libro que es llamado thesoro que fabla de todas las noblezas de las cosas" (5r). El texto queda interrumpido (folio 70r) en el capitulo 68 "Del desdén" (p. 214 columna a en la edición de Baldwin). En esta parte final faltan los títulos de los capítulos. 
sienpre dura" al menos en cuatro ocasiones (II.7.2; II.7.3; II.7.5.; II.7.6; Baldwin 95b-96a; Colombina 32va-b); "feliçidat que quiere dezir bondat acabada" (II.13.2; Baldwin 98a; Colombina 33va); "la bien andança que siempre ha de durar" (II.18.18; Baldwin 101a); "felicidad, que es bien andança perdurable (II.47.1; Baldwin; 121a; Colombina 40vb); "beatitudo, que es la bien andança perdurable" (II.126.1; Baldwin 173b; Colombina 56rb); "felicidad que es verdadera bien andanza" (Baldwin 122a, etc.).

- "moralidat que es dicha de buenas costumbres" (II.9.1; Baldwin 96b; Colombina 33a); "moralidat que quiere dezir de buenas costumbres" (II.10.3; Baldwin 97a; Colombina 33a; Y en II.50.1; Baldwin 123b; Colombina 41ra). "Virtud moral que quiere dezir de buenas costumbres" (II.46.1; Baldwin 119b; Colombina 39vb). Sólo aparece dos veces más sin esta coletilla.

- "abito tanto quiere dezir commo bondat o maldad que tiene onbre raygada en el coraçon \& que nunca la puede perder" (II.11.2; Baldwin 97b, Colombina 33rb). Y de forma similar: obras de abito, que son raygadas en el hombre..." (II.18.31; Baldwin 102a; Colombina 34va); abito que es cosa raygada en el coraçon del onbre" (II.18.31; Baldwin 102a; Colombina 34vb); "abito que es cosa raygada" (II.19.1; Baldwin 102a; Colombina 34vb); y "un abito raygado en el coraçon del onbre" II.41.1; Baldwin 113b, Colombina 38ra. Aparece otras 13 veces sin coletilla.

- "pasion, que quiere dezir sufrimiento" (II.14.1; Baldwin 98b; Colombina $33 \mathrm{va}$ ); las pasiones que son dichas sufrimientos (II.16.10 y II.17.1; Baldwin 99b; Colombina 33vb). Aparece varias veces más y sólo una vez más, como la pasión de Cristo.

-onbre sin escuela, que es dicho sin ensenamiento (II.16.8; Baldwin 99b; Colombina 33vb). Sólo aparece una vez.

-consideraçiones que son pensamientos para tomar el bien (II.17.4; Baldwin 100a; Colombina 34ra). Sólo aparece una vez.

- "tirano que quier decir señor cruel" (II.18.4; Baldwin 100b; Colombina 34ra). Aparece un total de siete veces el término pero sólo se aclara una vez. En las Partidas II.I.10 se define este término con las mismas palabras: "tirano tanto quiere dezir commo señor cruel que es apoderado en algunt rreyno o tierra por fuera [sic], o por enganno o por trayçion..."

-"elección que es escogimiento de voluntad" (II.18.8; Baldwin 100b, Colombina 34rb); "elecçiones que es escoger el bien o el mal" (II.18.10; Baldwin 101a, Colombina 34rb; aunque esta parte se encuentra en el original francés pocas líneas más abajo); "elección que quiere dezir escogimiento" (II.30.1; Baldwin 109a, Colombina 36vr) Aparece otras dos veces sin la aclaración, pero en el mismo párrafo.

- "opinión que quiere decir asmança" (II.18.11; Baldwin 101a, Colombina 34rb). Aparece unas diez veces en total, pero sólo en la segunda vez se explica su sentido.

- "la tercera manera de fuerça es por furia que es dicha sandez" (II.19.5; Baldwin 102b. En Colombina 34vb aparece: "la tercera manera de fortaleza es perfidia que es dicha sandez"). Sólo aparece una vez en el libro 2.

-prodigo, que espiende lo suyo sin recabdo (II.21.6; Baldwin 104a; Colombina 35rb)

-"Parvificus que quiere decir mendigo" (II.22.5; Baldwin 104b; Colombina 35rb). Sólo aparece una vez.

- "magnanimo es dicho onbre de grand coraçon" (II.23.1; Baldwin 104b; Colombina 35rb). En este caso se repite la definición ya traducida en Baldwin 99a. 
El término aparece al menos diez veces y profusamente en su forma sustantiva por lo que no vuelve a necesitar aclaraciones.

-"yracundo que quiere decir muy sañudo" (II.24.1; Baldwin 105b; Colombina 35vb) (sólo aparece una vez lo que quizás justifique esta explicación por lo demás innecesaria dado que el propio texto a continuación lo define)

-"artes mecánicas que son obras de mano" (II.44.22; Baldwin 117a; Colombina 39rv). No aparece más veces.

-"los poetas que son los sabios versyficadores" (II.45.2; Baldwin 118b; Colombina 39va).

-las cosas que son relativas, que quiere dezir cuando una cosa non puede ser sin la otra (II.46.5; Baldwin 120a, Colombina 40ra).

-el alma intelectual, que quiere dezir por que el onbre entiende (II.46.6; Baldwin 120a, Colombina 40ra). El término "entellectuel” en francés no siempre es traducido como pasa en II.7.8.

- "doctrina que quiere dezir enseñamiento" (II.48.3; Baldwin 122b; Colombina 40vb). El término sólo aparece unas tres veces. En el siglo XV se van a registrar los dos emparejados ("ciertas doctrinas provechosas para la doctrina y enseñamiento de los mancebos" en el prólogo de las Sentencias sobre amor, de Francisco López de Villalobos).

- "perpetuidad (perpetualidat) que quiere dezir que dure sienpre" (II.104.7; Baldwin 161b; Colombina 52va). Sólo aparece una vez. "Permanencia que es cosa firme \& estable" (II.42.1; Baldwin 114a, Colombina 38rb).

En otras ocasiones, se producen amplificaciones del contenido - como es el caso en: "el señorio del tirano es quando el señor cata tan solamente aquellos que a el aprovecha non catando aquello que es pro de su pueblo \& es contrario..." (II.44.1; Baldwin 115b, Colombina 38vb) - , que no son muy frecuentes y que no hemos contemplado en este estudio.

\section{INDICE B}

A continuación proporcionamos una lista de términos que a veces no son traducidos y cuando lo son, se emplean distintos términos. Para localizarlos, damos el número del libro, seguido del capítulo y del artículo:

-tabellion (secretario): traducido por tabeliones (III.77.3; III.79.8) y por notario (III.81.3; III.87.6)

-covenances (condiciones planteadas a aquel a quien se le ofrece el gobierno de la ciudad): es traducido como posturas (III.76.3), aposturas (III.74.3), pleitesías (III.79.2), convenencias (III.78.1)

-devanciers (los que preceden en recibir al señor): no es traducido

-senescal: senescal (III.79.8, 9) y mayordomo (III.80.2; III.94). En las

Partidas II,9,17 aparece definido como "ofiçial syn el qual non se debe fazer despensa en casa del Rey; e aun le llaman los antiguos asy, porque senes en latin tanto quiere dezir commo viejo, por rrazon que tiene ofiçio onrrado, e cuenta los omnes con piedras con que contaban. E por ende tanto muestra este nonbre commo ofiçial onrrado sobre las cuentas".

-poesté (señor de la ciudad, elegido por un año; Messelaar lo define como: "en Italie, gouverneur d'une cité, élu para celle-ci pour un an sous certaines conditions, soumis aux lois, assisté par deux conseils et tenu de rendre compte de son gouvernement, p. 5): potestad (a veces) y señor (III.82.8; III.81.1). En las Partidas II 1,13 aparece la definición de potestades - "e potestades llaman en Ytalia a los que 
escogen por regidores de las villas e de los grandes castillos, e estos an poder de juzgar segunt ley o fuero en aquellos lugares e sobre que son escogidos, en aquellas cosas e por tanto tienpo commo les fuere otorgado por los omnes daquel lugar, e non en mas" (443) - lo que indica que su sentido no era desconocido. ¿Quizás el propio Latini proporcionó esta definición?

-chapitres: artículos, fueros (III.83.1)

-commune: comunidad (I.44.1), común (III.77.1; III.78.4; III.81.3); concejo

(III.81.2), los de aquel logar (III.80.1).

-prouvost (señor de la ciudad, nombrado por un año): justicia (III.73.4);

señor (III.75.15); prebeste (III.76.1); preboste (III.104.3)

-provosté (dirección suprema de la ciudad, cargo de prouvost): señorío (III.76.4), proboste (III.101.t).

-eschievin (magistrado unido al alcalde escogido por un año): traducido como "los otros oficiales" (III.73.4).

Fecha de recepción del artículo: diciembre de 2009.

Fecha de aceptación y versión final: septiembre de 2010. 